\title{
Detección del gen de fusión CBFB-MYH11/INV (16) en paciente con leucemia aguda mielomonocítica
}

\section{Detection of the CBFB-MYH11 / INV (16) fusion gene in a patient with acute myelomonocytic leukemia}

\author{
Manuel Alfredo Campoverde Cisneros ${ }^{1,3,4}$, Héctor Chiang Wong ${ }^{2}$ y Mauro Javier Arcentales Cayamcela ${ }^{3}$ \\ ${ }^{1}$ Universidad Católica de Cuenca, Ecuador \\ 2 Servicio de Hematología del Hospital José Carrasco Arteaga, Cuenca, Ecuador \\ ${ }^{3}$ Laboratorio de Diagnóstico Molecular de Alta Especialidad BIONCOGÉN, Cuenca, Ecuador \\ ${ }^{4}$ Sociedad de Hematología Núcleo del Austro (SEHNA), Ecuador \\ *manuel.campoverde@ucacue.edu.ec
}

DOI: https://doi.org/10.26871/killkana_salud.v4i2.643

\begin{abstract}
Resumen
Contexto: La leucemia aguda mielomonocítica es un subtipo de leucemia mieloide aguda por la presencia de la inversión del cromosoma 16 que provoca la formación de un gen de fusión denominado CBFB-MYH11. Objetivo: Detectar la presencia de este gen fusion es crítico en el diagnóstico de esta patología. Con este caso se busca consolidar la implementación de una prueba molecular altamente sensible y específica para la detección del gen CBFB-MYH11. Materiales y Métodos: A partir de sangre periférica se extrajo el ARN del paciente diagnosticado con leucemia mieloide aguda. Se realizó RT-PCR anidada para la detección de CBFB-MYH11 adaptado al protocolo preestablecido. Resultados: Se acoplo y estandarizo el método para detectar mediante RT-PCR el gen de fusion CBFB-MYH11. Mediante esta técnica se logró identificar la inversión del cromosoma 16 en el caso de estudio. Conclusiones: El resultado coincide con otras investigaciones previas. La detección de esta y otras aberraciones cromosómicas mediante pruebas moleculares permite tomar decisiones terapéuticas cruciales para mejorar el pronóstico y la calidad de vida del paciente.
\end{abstract}

Palabras clave: Leucemia Mieloide Aguda, adultos, CBFB-MYH11, inversión cromosoma 16.

\begin{abstract}
Context: Acute myelomonocytic leukemia is an acute myeloid leukemia subtype due to the inversion of chromosome 16 that causes the formation of a fusion gene called CBFB-MYH11 Objective: Detecting the existence of this fusion gene is critical in the diagnosis of this pathology. This case seeks to consolidate the implementation of a highly sensitive and specific molecular test for the detection of the CBFB-MYH11 gene. Methodology: RNA of the patient diagnosed with acute myeloid leukemia, was taken from peripheral blood. A nested RT-PCR, adapted to the pre-established protocol, was performed to detect CBFB-MYH11. Results: The method for detecting the fusion gene CBFB-MYH11 was adjusted and standardized by using RT-PCR. This technique allowed to identify chromosome 16 inversion in the case study. Conclusions: The result is in line with other previous research studies. Detecting this and other chromosomal aberrations, by molecular testing, allows to make crucial therapeutic decisions for the patient's prognosis and quality of life improvement.
\end{abstract}

Keywords: Acute Myeloid Leukemia, Adults, CBFB-MYH11, Chromosome 16 inversion.

\section{Introducción}

En la génesis de la leucemia mieloide aguda (LMA) existen causas genéticas que involucran las células precursoras mieloides originando alteraciones en la función, diferenciación, multiplicación y posterior acumulación en la medula ósea (MO) y sangre periférica. ${ }^{1}$ Los casos de LMA en el mundo presentan la incidencia de 3.7 por 100.000 habitantes y su incremento está relacionado con la edad avanzada de la población, otro índice es la tasa de mortalidad del $1.2 \%$ con predominio del sexo masculino, está patología en relación a todos los tipos de leucemia agudas ostenta la frecuencia en adultos del $80 \%$ y en la edad pediátrica del $15-20 \%$. $^{2,3}$ En el Ecuador se reportó según el Registro Nacional de Tumores de SOLCA Núcleo de Quito la LMA independiente del tipo de la línea celular, ocupa en la escala el sexto lugar en hombres y el séptimo en mujeres; un estudio descriptivo realizado en Hospital Solca Núcleo Quito durante 4 años proporcionó información 
sobre la edad media de los pacientes es de 33 años siendo representativo el sexo masculino., ${ }^{4,5}$

La característica de un pronóstico favorable de recuperación y la frecuencia aproximada de la inversión (inv.) del cromosoma 16 (p13.1q22) es del $4 \%$ de todos los casos en la LMA (Tabla 1),está alteración estructural en el cromosoma 6 genera un gen de fusión entre CBFB (subunidad beta del factor de unión al núcleo) en posición $16 q 22$ por la secuencia 5' 'y MYH11(cadena pesada de miosina de musculo liso 11) en posición 16p13.1 con la secuencia 3' (Figura 1), lo que origina una proteína quimérica que inicia la leucemogénesis al inhibir la diferenciación celular. ${ }^{6-9}$

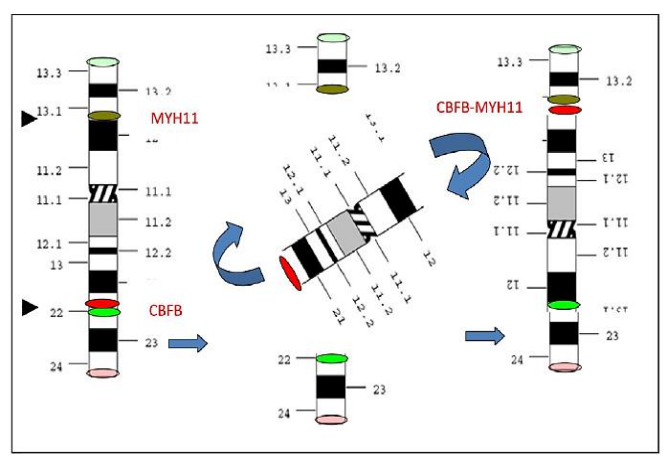

Fig. 1: Representación esquemática del gen de fusión CBFB-MYH11, inv. (16) (p13q22)

Fuente: Poddighe PJ, et al. A novel cryptic CBFB-MYH11 gene fusion present at birth leading to acute myeloid leukemia and allowing molecular monitoring for minimal residual disease. Hum Pathol Case Rep.

Tabla 1: Clasificación pronóstica según citogenética y estado mutacional

\begin{tabular}{|c|c|}
\hline Risk category ${ }^{\star}$ & Genetic abnomality \\
\hline Favorable & $\begin{array}{l}\mathrm{t}(8 ; 21)(\mathrm{q} 22 ; \mathrm{q} 22.1) ; \text { RUNX1-RUNX1T1 } \\
\text { inv(16)(p13.1q22) or } \mathrm{t}(16 ; 16)(\mathrm{p} 13.1 ; \mathrm{q} 22) ; C B F B-M Y H 11 \\
\text { Mutated NPM1 without FLT3-ITD or with FLT3-ITD }{ }^{\text {low }} \dagger \\
\text { Biallelic mutated CEBPA }\end{array}$ \\
\hline Intermediate & $\begin{array}{l}\text { Mutated NPM1 and FLT3-ITD }{ }^{\text {high }} \dagger \\
\text { Wild-type NPM1 without FLT3ITD or with FLT3-ITD }{ }^{\text {low }} \dagger \text { (without } \\
\text { adverse-risk genetic lesions) } \\
\mathrm{t}(9 ; 11)(\mathrm{p} 21.3 ; \mathrm{q} 23.3) ; \text { MLLT3-KMT2A } \ddagger \\
\text { Cytogenetic abnormalities not classified as favorable or adverse }\end{array}$ \\
\hline Adverse & 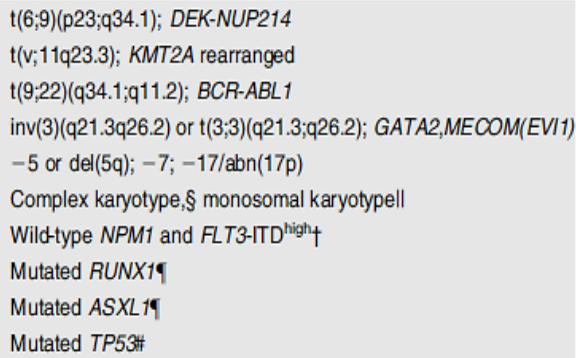 \\
\hline
\end{tabular}

Fuente: Döhner $H$ et al. Diagnosis and management of AML in adults: 2017 ELN recommendations from an international expert panel. Blood 2017; 129(4): 424-47.
La detección de la inv. (16) es común en pacientes con el subtipo M4 de LMA (LMAM4) según la clasificación Franco-Estadounidense (FAB) (Tabla.2) y la Organización Mundial de la Salud establece ocho categorías (OMS) (Tabla.3). Los parámetros citomorfológicos reportan poblaciones de blastos mieloides, monoblastos y celulas monocitoides atípicas. ${ }^{10-12}$

\section{Clasificación LMA-FAB}

\begin{tabular}{|c|c|}
\hline Subtipo & Nombre \\
\hline M0 & Leucemia mieloide aguda indiferenciada \\
\hline M1 & $\begin{array}{l}\text { Leucemia mieloide aguda con maduración } \\
\text { mínima }\end{array}$ \\
\hline $\mathrm{M} 2$ & Leucemia mieloide aguda con maduración \\
\hline M3 & Leucemia promielocítica aguda \\
\hline M4 & Leucemia mielomonocítica aguda \\
\hline M4eos & Leucemia mielomonocítica aguda con eosinofilia \\
\hline M5 & Leucemia monocítica aguda \\
\hline M6 & Leucemia eritroide aguda \\
\hline M7 & Leucemia megacarioblástica aguda \\
\hline
\end{tabular}

Fuente: Cruz-Santana et al. Observaciones relacionadas con losmetodos diagnosticos ideales en el paciente con leucemiamieloide aguda. Rev. Hemotol. 2016; 17(3):187-194

\section{Clasificación LMA-OMS}


Fundamentados en las últimas recomendaciones del grupo de expertos de European Leukemia Net (ELN 2017) y las aprobaciones recientes de drogas-target por los organismos reguladores, el panel genético-molecular básico sugerido debería englobar al ${ }^{13-16}$ FLT3-ITD por su implicación pronóstica (aunque existen algunas divergencias casi todos los estudios difundidos hasta la fecha coinciden en otorgar un papel pronóstico adverso) y terapéutica:, ${ }^{17,18}$ el NPM1 desde el punto de vista de la evolución de los pacientes, la mayoría de trabajos conceden un papel favorable, ${ }^{19} \mathrm{CEBPA}$ (bialélico) se pretrende relacionarse con mejor pronóstico, también está ligada dentro del subgrupo de casos vinculados a predisposición de línea germinal (familiar),TP53ASXL1 asociados a mal pronóstico y RUNX1 relacionado a mal pronóstico tambien si coexiste con anormalidades genéticas favorables se consideraría como de buen pronóstico. ${ }^{13}$

Para el diagnóstico inicial y definitivo de la LMAM4 se requiere información de la anamnesis, análisis-exámenes de sangre y medula ósea con reportes citomorfológicos, inmunofenotipificación, citogenética, pruebas moleculares. Actualmente la secuenciación de próxima generación (NGS) aporta con información proveniente de mutaciones puntuales. ${ }^{20,21}$ Una de las pruebas moleculares que con frecuencia se ejecuta en el diagnostico, pronostico y monitorización es la Reacción en Cadena de Polimerasa Transcriptasa Inversa (RT-PCR) anidada, es una técnica semi cuantitativa que utiliza el ADN (sangre periférica o MO) complementario a partir de ARN para la identificación de genes, haciendo posible establecer el fenotipo de la leucemia. ${ }^{22,23}$

\section{Caso Clínico}

Mujer de 57 años con disnea y equimosis en extremidades inferiores, antecedentes de miastenia graves e hipertensión arterial, se realiza análisis y exámenes primarios de sangre periférica (Tabla 4).

Tabla 4: Análisis y exámenes primarios de sangre periférica

\begin{tabular}{|c|c|c|c|c|c|}
\hline \multicolumn{2}{|c|}{ Hemograma. } & \multicolumn{2}{|c|}{ Química sanguínea. } & \multicolumn{2}{|c|}{ Hemostasia. } \\
\hline Leucocitos. & $27.340 \mathrm{cel} / \mathrm{mm}^{3}$ & Sodio. & $137 \mathrm{meq} / \mathrm{L}$ & TTPa. & 33.9Seg. \\
\hline Neutrófilos. & $1.720 \mathrm{cel} / \mathrm{mm}^{3}$ & Creatinina. & $1.11 \mathrm{mg} / \mathrm{dl}$ & INR. & 1.39 \\
\hline Monocitos. & $16.060 \mathrm{cel} / \mathrm{mm}^{3}$ & Albúmina. & $4 \mathrm{~g} / \mathrm{dl}$ & Ser & gía. \\
\hline Hemoglobina. & $4.9 \mathrm{mg} / \mathrm{dl}$ & Proteínas Totales. & $5.4 \mathrm{~g} / \mathrm{dl}$ & Hepatitis B. & \\
\hline Plaquetas. & $4.000 \mathrm{cel} / \mathrm{mm}^{3}$ & Bilirrubina Total & $0.61 \mathrm{mg} / \mathrm{dl}$ & Hepatitis C. & Negativo. \\
\hline ALT & $6 \mathrm{U} / \mathrm{L}$ & Bilirrubina directa. & $0.17 \mathrm{mg} / \mathrm{dl}$ & VIH & \\
\hline LDH & $572 \mathrm{U} / \mathrm{L}$ & AST. & $15 \mathrm{U} / \mathrm{L}$ & PCR. & $19 \mathrm{mg} / \mathrm{dl}$ \\
\hline
\end{tabular}

Fuente: Los autores

cel/mm3: celulas por milímetro cubico, $\mathbf{m g} / \mathbf{d l}$ : miligramos por decilitro, meq/L: mili equivalente por litro, g/dl: gramos por decilitro, $\mathbf{U} / \mathbf{L}$ : unidades por litro, seg: segundos

Citomorfología de Aspirado de Médula Ósea (AMO): hipercelularidad, grasa disminuida. Megacariocitos disminuidos en número con morfología normal. Se observa una infiltración por promonocitos $(10 \%)$ y blastos $(26 \%)$ de mediano y gran tamaño, núcleo de contorno regular, con frecuencia con forma arriñonada (monocitoide). La serie eritroide se encuentra bien representada (24\%) en todos sus estadios de maduración. La serie mieloide con presencia de todos los precursores mieloides. (30\%). Linfocitos (3\%), células plasmáticas (1\%). (Figura 2 y 3).

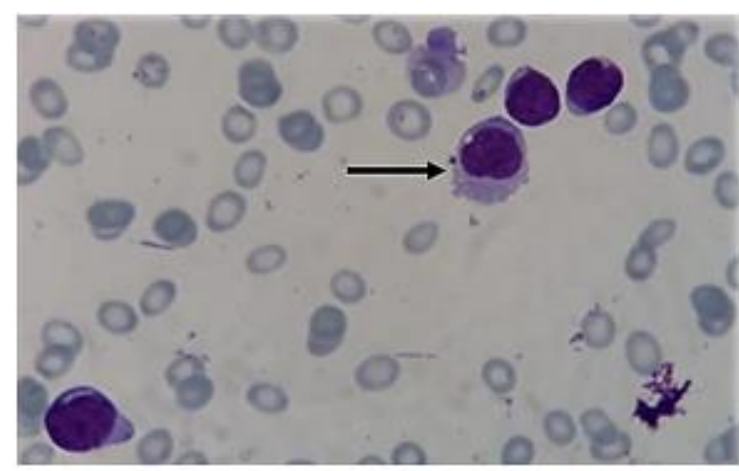

Fig. 2: Blastos de mediano y gran tamaño, núcleo de contorno regular, con frecuencia con forma arriñonada (monocitoide)

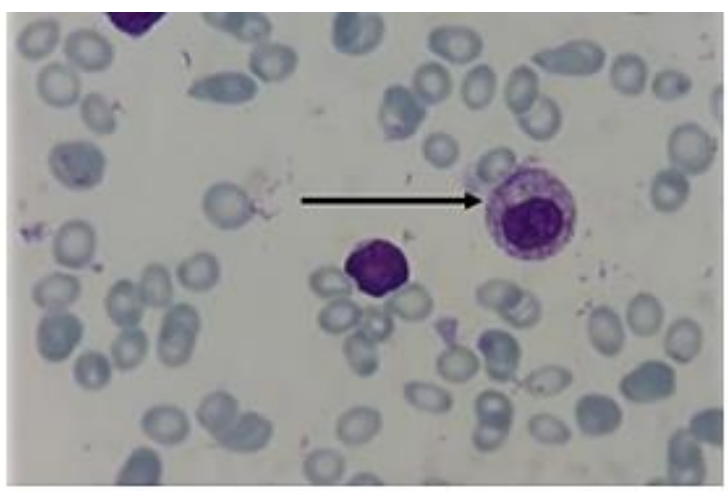

Fig. 3: Presencia de precursores mieloides y neutrófilos mayores del $20 \%$

Citometría de Flujo en Medula Ósea: se evaluaron 200.000 eventos con el $90 \%$ de células viables. La curva de maduración de la serie granulocito-neutrófilos se encuentra alterada, sólo se observan formas maduras. Se detectan dos poblaciones patológicas por inmunofenotipo, la población 1 corresponden fenotípicamente a células de la serie monocitoide y la población 2 constituye blastos con fenotipo de estirpe mieloide (Tabla 5); se detectó una población de blastos mieloides en presencia del $48.34 \%$ de promonocitos y monocitos, compatible con Leucemia Mielomonocítica Aguda (LMA M4) 
Tabla 5: Diagnóstico por inmunofenotipo

\begin{tabular}{|l|l|l|l|}
\hline \multicolumn{2}{|c|}{ POBLACION 1 (35,97\%) } & \multicolumn{2}{c|}{ POBLACION 2 (48,34\%) } \\
\hline CD13+D & CYTCD3- & CD13+ & CYTCD3- \\
\hline CD15D+ & CD3-; CD7- & CD14+; CD15+ & CD3-; CD7- \\
\hline CD33+D & CD10-; & CD33+ & CD10- \\
\hline CD34+ & CD11B- & CD11B+ & CD34- \\
\hline CD38+ & CD14-; CD16- & CD35+; CD36+ & CD16- \\
\hline CD45+ & CD19-; CD35- & CD38+D; CD45+ & CD19- \\
\hline CD117+ & CD36-; CD64- & CD64+ & CD117- \\
\hline CYTMPO+ & CYTCD79A- & CYTMPO+ & CYTCD79A- \\
\hline HLADR+ & NTDT- & HLADR+ & NTDT- \\
\hline
\end{tabular}

Fuente: Los autores

\section{Citogenética Convencional MO.}

- CARIOTIPO: 46 XX, se analiza 20 metafases mediante bandas GTG, que son citogenéticamente normales.

Biología molecular en Sangre periférica por RT-PCR.

- Análisis negativo para: FLT3/ITD, PLM/Rara, AML1ETO, BCR/ABL.

- Análisis positivo para: CBF-MYH11/Inversión del cromosoma 16 (inv. (16) (p13.1q22) B.

\section{PCR para detección del gen fusión CBF/MYH11.}

La extracción del ARN se obtuvo por la Técnica Trizol (fenol-cloroformo y guanidina tiocianato) descrita por Chomczyski y en la obtención del ADN complementario se empleó el kit Script Reverse Transcriptasa. ${ }^{24}$

El proceso de amplificación por RT-PCR anidad se emplearon los pares de cebadores CBFB y MYH11 (Tabla 6).

Tabla 6: Diagnóstico por inmunofenotipo

\begin{tabular}{|c|c|}
\hline Primer code & \multicolumn{1}{|c|}{ Sequence $\mathbf{5}^{\circ}$-- $\mathbf{3}^{-}$} \\
\hline CBFB-A & GCA GGC AAG GTA TAT TTG AAG G \\
\hline MYH11-B1 & TGA AGC AAC TCC TGG GTG TC \\
\hline MYH11-B2 & TCC TCT TCT CCT CAT TCT GCT C \\
\hline CBFB-C & GGG CTG TCT GGA GT TGA TG \\
\hline MYH11-D1 & TCC CTG TGA CGC TCT CAA CT \\
\hline MYH11-D2 & CT GAG CGC CTG CAT GT \\
\hline
\end{tabular}

Fuente: Dongen J van et al. Standardized RT-PCR analysis of fusion gene transcripts from chromosome aberrations in acute leukemia for detection of minimal residual disease. Leukemia . 1999; 13(12): 190128.

Los productos amplificados son analizados cualitativamente por electroforesis en gel de agarosa al $2 \%$ utilizando bromuro de etidio como colorante de revelado.

En la primera PCR se amplifico el producto con los cebadores A-B2, la mutación se identificó a de 418 pb como observamos en la Figura 4.

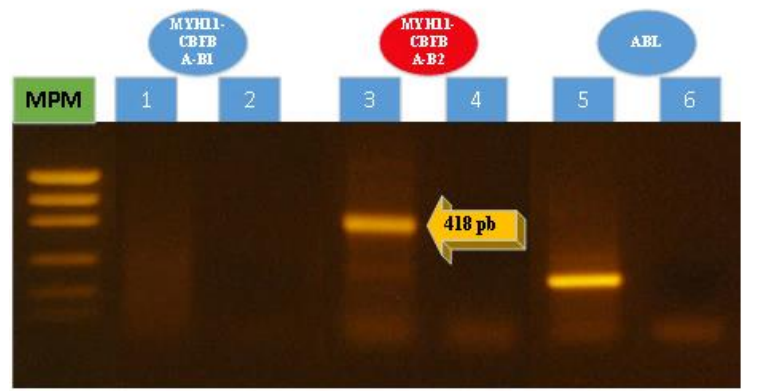

Fig. 4: Primera amplificación del gen de fusion MYH11/CBFB (1= A-B1; 3=A-B2), 5 ABL, 2-4-6 controles negativos. MPM: marcador de peso molecular de $100 \mathrm{pb}, \mathrm{pb}$ : pares de bases

En la segunda PCR se amplifico el producto con los cebadores C-D2, la mutación se identificó a de 271 pb como observamos en la Figura 5.

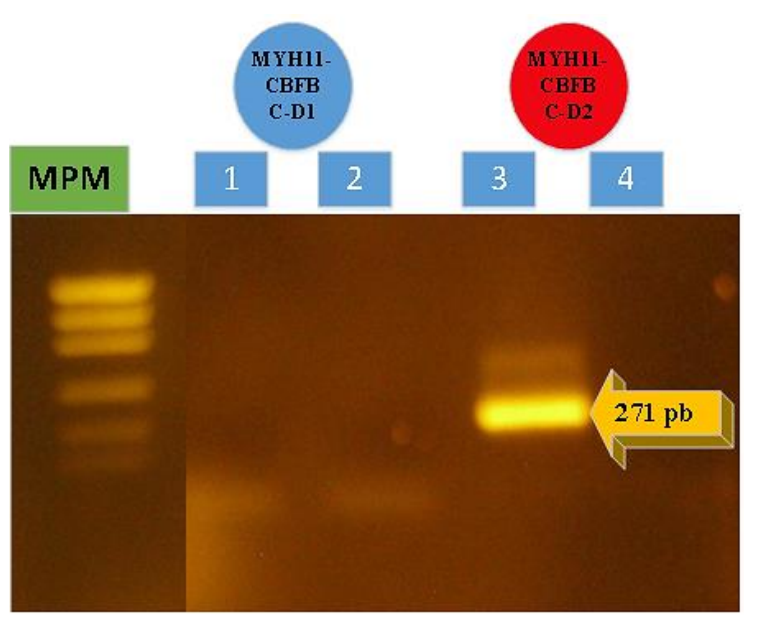

Fig. 5: Segunda amplificación de MYH11/CBFB (1= C-D1; 3=C-D2), 2-4 controles negativos. MPM: marcador de peso molecular de 100pb, pb: pares de bases

\section{Tratamiento de primera linea.}

La LMA con inv. (16) (p.13.1q22) según la clasificación de la OMS 2016 se categorizada como de pronóstico favorable. Se inició el tratamiento bajo protocolo LMAMD ANDERSON con esquema específico para el ciclo de inducción, ciclo de consolidación y CTF en AMO (Tabla 7). 
Tabla 7: Tratamiento de inducción y post-remisión en el paciente

\begin{tabular}{|c|c|c|c|c|c|}
\hline \multirow[b]{3}{*}{ ESQUEMA } & \multirow{2}{*}{\multicolumn{2}{|c|}{ CICLO DE INDUCCION: }} & \multicolumn{3}{|c|}{ CICLO DE CONSOLIDACION } \\
\hline & & & \multicolumn{2}{|c|}{$\begin{array}{c}\text { 1ra } \\
\text { intensificación } \\
\end{array}$} & 2da intensificación \\
\hline & \multicolumn{2}{|c|}{$\begin{array}{l}\text { IDARRUBICINA/ } \\
\text { CITARABINA }(3+7)\end{array}$} & \multicolumn{2}{|l|}{$\frac{\text { CITARABINA/ }}{\text { ETOPOSIDO }}$} & $\begin{array}{l}\text { CITARABINA/ } \\
\text { IDARRUBICINA }\end{array}$ \\
\hline \multirow{7}{*}{ AMO } & $\begin{array}{l}\text { Granulocitos/ } \\
\text { Neutrófilos }\end{array}$ & $68.7 \%$ & \begin{tabular}{|l|l}
$\begin{array}{l}\text { Granulocitos/ } \\
\text { Neutrófilos }\end{array}$ \\
\end{tabular} & $82.2 \%$ & \multirow{9}{*}{$\begin{array}{l}\text { Actualmente en } \\
\text { evolución }\end{array}$} \\
\hline & mieloblastos & $1.82 \%$ & mieloblastos & $2.1 \%$ & \\
\hline & $\begin{array}{l}\text { Promielocitos } \\
\text { mielocitos }\end{array}$ & $(14.56 \%)$ & Promielocitos y mielocitos & $21.9 \%$ & \\
\hline & metamielocitos & $35.7 \%$ & metamielocitos & $47.5 \%$ & \\
\hline & Bandas y neutrófilos & $47.8 \%$ & Bandas y neutrófilos & $27.6 \%$ & \\
\hline & $\begin{array}{l}\text { Monocitos/células } \\
\text { dendríticas }\end{array}$ & $12.7 \%$ & $\begin{array}{l}\text { Monocitos/células } \\
\text { dendríticas }\end{array}$ & $5.30 \%$ & \\
\hline & $\begin{array}{l}\text { Linfocitos totales } \\
+\mathrm{NK}\end{array}$ & $14.7 \%$ & Linfocitos totales $+\mathrm{NK}$ & $14.7 \%$ & \\
\hline \multicolumn{2}{|c|}{5.000 .000 de eventos/vialidad } & $88 \%$ & $\begin{array}{l}2.000 .000 \mathrm{de} \\
\text { eventos/vialidad }\end{array}$ & $80 \%$ & \\
\hline \multicolumn{2}{|c|}{$\begin{array}{l}\text { Compromiso madurativo hacia línea } \\
\text { mieloide }\end{array}$} & $1.61 \%$ & Eosinófilos/ total celular & $0.05 \%$ & \\
\hline \multicolumn{3}{|c|}{$\begin{array}{l}\text { Serie granulocito/neutrófilos se encuentra alterada } \\
\text { en los estadios IV }\end{array}$} & \multicolumn{3}{|c|}{$\begin{array}{l}\text { Serie granulocito/neutrófilos se encuentra alterada en los } \\
\text { estadios II, III y IV }\end{array}$} \\
\hline \multicolumn{3}{|c|}{ Serie monocítica / normal } & \multicolumn{3}{|c|}{ Serie monocítica / normal } \\
\hline \multirow{2}{*}{\multicolumn{3}{|c|}{\begin{tabular}{|l|} 
Linfocitos B: no hay variación \\
Serie roja se encuentra disminuida
\end{tabular}}} & \multicolumn{3}{|c|}{ Linfocitos B: no hay variación } \\
\hline & & & & & \\
\hline \multicolumn{3}{|c|}{$\begin{array}{l}\text { MO por CMF no se detecta proliferación clonal o } \\
\text { actividad por LMA. }\end{array}$} & \multicolumn{3}{|c|}{$\begin{array}{l}\text { MO por CMF es negativo para infiltración por células mieloides } \\
\text { o monocitoides }\end{array}$} \\
\hline
\end{tabular}

\section{Discusión}

En este trabajo se detectó por RT-PCR anidad el gen de fusión $\mathrm{CBF} \beta / \mathrm{MYH} 11$ en paciente con diagnóstico de LMA M4 con inv. ${ }^{16}$ (p13.1q22) según la última clasificación de la WHO 2016. ${ }^{13,25}$ Como agregado presentaba Miastenia Gravis (conocido previamente), por lo que durante toda su evolución se mantuvo con su medicación de base (piridostigmina, micofenolato de mofetil) y se limitó el uso de ciertos fármacos por su riesgo a exacerbar una crisis miasténica.

La integración de los marcadores citogenéticos y moleculares ha innovado la estratificación pronóstico (favorable, intermedio, alto riesgo) de la Leucemia Mieloide Aguda (LMA) simplificando el desarrollo del concepto de terapia adaptada al riesgo. ${ }^{13,26}$

Tanto la t $(8 ; 21)$ y la inv.(16) son alteraciones citogenéticas que se asocian a pronóstico favorable, ambas tienen en común que resultan en dos transcritos (AML1/ETO y $\mathrm{CBF} \beta / \mathrm{MYH} 11$ respectivamente) que engloban los genes encargados de la codificación de los dos heterodímeros del "core binding factor" (CBF), $\mathrm{CBF} \alpha$ y $\mathrm{CBF} \beta$. Estos tienen un riesgo de recidiva menor al observado en los grupos de riesgo intermedio y alto; y parece que se benefician especialmente del tratamiento con altas dosis de citarabina durante la consolidación; ${ }^{26,27}$ el caso de nuestra paciente, por todo lo previamente expuesto fue categorizada como de pronóstico favorable, pero sin la posibilidad de realizar un panel molecular ampliado (accesibilidad, costo, disponibilidad, secuenciación masiva, etc.) que nos permita hacerlo con mayor certeza.

Existen estudios en donde al detectar marcadores moleculares como FLT3 (FLT3-ITD), NPM1, o CEBP $\alpha$ ha permitido mejorar la estratificación pronóstico de los pacientes con cariotipo normal y se hace necesaria en la actualidad para el manejo clínico de este subgrupo de enfermos en cuanto al riesgo estimado de recidiva y la supervivencia libre de enfermedad. ${ }^{13,17,18}$
Debido a que los nuevos agentes ofrecen limitados mejoramientos en cuanto a supervivencia global, el trasplante alogénico persiste siendo el gold standard para aquellos que pueden tolerar el procedimiento y en quienes no adquieran la remisión postquimioterapia ${ }^{15}$

\section{Conclusiones}

El uso de la técnica de RT-PCR anidada en el diagnóstico de la Leucemia Aguda Mielomonocítica mediante detección del punto de rotura del gen de fusión $\mathrm{CBF} \beta / \mathrm{MYH} 11$ permite tomar decisiones terapéuticas cruciales para mejorar el pronóstico y la calidad de vida del paciente.

\section{Recomendaciones}

De forma aplicable a nuestro medio, es muy importante seguir enfatizando la transcendencia de la biología molecular dentro del campo de LMA; por lo que es esencial seguir ampliando los paneles moleculares que nos integre mejor las resoluciones con el paciente.

\section{Fuente de Financiamiento}

Este estudio es autofinanciado.

\section{Conflicto de Intereses}

No existen conflictos personales, profesionales, financieroso de otro tipo.

\section{Consentimiento Informado}

Los autores cuentan con el consentimiento informado de los pacientes para la investigación, la publicación del caso y sus imágenes.

\section{Referencias Bibliográficas}

1. Anci Alvarez C, Hoffmann M, Gisbert P, Al E. Evaluación de pacientes con leucemia mieloide aguda. Experiencia institucional. Hematologia. 2019;23(1):8-12.

2. Quintero Sierra Y, Hernández Padrón C, Romero González A, Fernández YC, Macia Pérez I, Llerena Moreno D, et al. Incorporación de las altas dosis de antraciclina en el tratamiento de la leucemia mieloide aguda del adulto. Revista Cubana de Hematología, Inmunología y Hemoterapia. 2019;35(1).

3. Pacheco L. Leucemia mieloide aguda - Revista de Hematología;

4. Gonzalez GM. Análisis de supervivencia global de pacientes diagnosticados con leucemia mieliode aguda en relación a factores de riesgo citogenético del Hospital Solca Quito durante el período mayo 2010 hasta mayo 2014; 2015.

5. Garrote Santana H, Amor Vigil AM, Díaz Alonso CA, Fernández Martínez L, Ruiz Moleón V, Machín García S, et al. Caracterización del gen de fusión 
RUNX1-RUNX1T1 en pacientes cubanos con leuce-19. Sayar H, Bashardoust P, Cripe L, Chakraborty S, Bai mia mieloide aguda, 2000-2016 TT. Rev cuba hematol inmunol hemoter. 2018 sep;34(3):1-16. AvaiS. Long-term outcome of NPM1 mutated acute myeloid leukemia: A single-institution experience; 2017.

lable from: http://www.revhematologia.sld.cu/index.php/20. Arber DA, Borowitz MJ, Cessna M, Etzell J, Foucar K, hih/article/view/910/799.

6. Opatz S, Vosberg S, Ksienzyk B, Tschuri S, Graf A, Krebs $\mathrm{S}$, et al. Clonal Evolution of Relapsed CBFB/MYH11 Rearranged Acute Myeloid Leukemia (AML). Blood. 2018 nov;132(Supplement 1):2772-2772.

Hasserjian RP, et al. Initial Diagnostic Workup of Acute Leukemia: Guideline From the College of American Pathologists and the American Society of Hematology. Archives of Pathology \& Laboratory Medicine. 2017 feb;141(10):1342-1393.

7. Saida S, Zhen T, Kwon EM, Lopez G, Liu PP. Distinct 21. Aypar U, Smoley SA, Pitel BA, Pearce KE, Zenka RM, Roles of GATA2 in Development and Evolution of CBFBMYH11 AML. Blood. 2018 nov;132(Supplement 1):770 770.

Vasmatzis $\mathrm{G}$, et al. Mate pair sequencing improves detection of genomic abnormalities in acute myeloid leukemia. European Journal of Haematology. 2019;102(1):87-96.

8. Kayser S, Levis MJ. Clinical implications of molecular 22. Garrote Santana H, Lavaut-Sánchez K, Amor Vigil AM, markers in acute myeloid leukemia; 2019.

9. Hyde RK, Zhao L, Alemu L, Liu PP. Runx1 is required for hematopoietic defects and leukemogenesis in CbfbMYH11 knock-in mice. Leukemia. 2015 aug;29(8):1771-23. 1778.

10. Wang ML, Bailey NG. Acute myeloid leukemia genetics risk stratification and implications for therapy. Archives of Pathology and Laboratory Medicine. 2015 sep;139(10):1215-1223.

Díaz Alonso C, Fernández Martínez L, Ruiz Moleón V, et al.. Cinco décadas de la biología molecular y la citogenética aplicadas a la hematología Cubana; 2017.

1. Amor Vigil AM, Hernández Miranda LL, Díaz Alonso 24 CA, Fernández Martínez L, Ruíz Moleón V, Garrote Santana H. La biología molecular en la precisión diagnósticade las leucemias TT. Rev cuba hematol inmunol hemoter. 2018;34(3):1-7. Available from: 25 http://scielo.sld.cu/scielo.php?script=sci_arttext\&pid= S0864-02892018000300007.

12. Lira V P. Leucemia aguda mieloide: diagnóstico y tratamiento. ARS MEDICA Revista de Ciencias Médicas. 26. 2017 may;18(4):95.

. Sasaki K, Kantarjian HM, Luthra R, Patel KP, GarciaManero G, Verstovsek S, et al. Qualitative and Quantitative Correlation of PML-Rara Fusion Transcript from Peripheral Blood and Bone Marrow Samples By Quantitative Real-Time PCR in Patients with Acute Promyelocytic Leukemia. Blood. 2015;126(23):3756-3756.

4. Cayamcela A, Javier M. Reacción en cadena de polimerasa transcriptasa inversa para diagnóstico de leucemia mieloide crónica Cuenca 2013-2017. 2018. Available from: http://dspace.ucuenca.edu.ec/handle/123456789/31323.

5. Arber DA, Orazi A, Hasserjian R, Thiele J, Borowitz MJ, Le Beau MM, et al.. The 2016 revision to the World Health Organization classification of myeloid neoplasms and acute leukemia; 2016.

13. Döhner H, Estey E, Grimwade D, Amadori S, Appelbaum 27. FR, Büchner T, et al.. Diagnosis and management of AML in adults: 2017 ELN recommendations from an international expert panel; 2017.

14. Pollyea DA. New drugs for acute myeloid leukemia inspired by genomics and when to use them. Hematology (United States). 2018;2018(1):45-50.

15. DeStefano CB, Hourigan CS. Personalizing initial therapy in acute myeloid leukemia: incorporating novel agents into clinical practice. Therapeutic Advances in Hematology. 2018 may;9(5):109-121.

16. Bewersdorf JP, Shallis R, Stahl M, Zeidan AM. Epigenetic therapy combinations in acute myeloid leukemia: what are the options? Therapeutic Advances in Hematology. 2019; 10:204062071881669.

17. Leick MB, Levis MJ. The Future of Targeting FLT3 Activation in AML; 2017.

18. Garcia JS, Stone RM. The Development of FLT3 Inhibitors in Acute Myeloid Leukemia; 2017.

Recibido: 3 de septiembre de 2019

Aceptado: 10 de mayo de 2020 cular biomarkers in acute myeloid leukemia; 2017.

Sinha C, Cunningham LC, Liu PP. Core Binding Factor Acute Myeloid Leukemia: New Prognostic Categories and Therapeutic Opportunities; 2015.

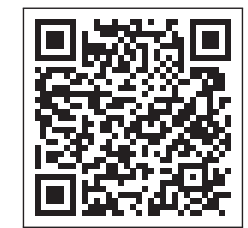

